\title{
Supplementary Comment on \\ "Investigation of Two Possible Compression Sites of the Deep Branch of the Radial Nerve and Nerve Supply of the Extensor Carpi Radialis Brevis Muscle"
}

This is a meticulous study of the anatomical relationships between the radial and posterior interosseous nerves on one side and the supinator muscle and its tendons and fibrous bands on the other. The variations were examined in 60(!) arms giving a considerable insight into possible damage in the form of radial and posterior interosseous syndromes, the main reason for the entrapments being the arcade of Frohse. There were no racial differences between the Turkish population and others. Although clinical implications and correlations were merely touched on, the study is very valuable for neurologists and surgeons dealing with therapy of peripheral nerve entrapment syndromes.

Ernst Heinrich GROTE, M.D. Abteilung für Neurochirurgie Universitätsklinikum Tübingen

Tübingen, Germany

This is a supplementary comment on an article published in Neurologia medico-chirurgica Vol. 44, No. 1, pp 14-19. 